\title{
GSM based Monitoring and Control of Systems
}

\author{
Saima Maqbool ${ }^{a^{*}}$, Sajaad Ahmed Lone ${ }^{\mathrm{b}}$ \\ ${ }^{a, b}$ Department of Computer Science and Engineering, \\ Islamic University of Science and Technology, Awantipura-192122, Pulwama, Jammu \& Kashmir, India
}

\begin{abstract}
In this paper we have proposed a method to make a base for the monitoring and Control of systems. The monitoring and Control of systems could be from monitoring of movements in any particular area to the monitoring of any external electrical, mechanical device. Every person is pocketed with the cell. So, taking the advantage of the same we developed the system for the monitoring and Control system using GSM technology. This will provide the owner, manager of the system to know about the working or any kind of default in the system. Also the system is controlled which is needed in special circumstances and is be implemented with the help of complete circuitry which is a different module (DTMF module) has a specific code as input through GSM encoder that is processed by decoder circuitry of our system. The goal is to find an improvement over the previous surveillance systems. The maintainability of the new and improved is quite efficient as it is easy to handle it by simple software demo. The facilities provided by it such as easy handling, a secrecy support, an immediate action are granted. A prototype is built in the Windows platform using C\# to demonstrate the functioning of the entire system.
\end{abstract}

Index Terms: GSM, Modem, C\#, Monitoring System, AT Commands, DTMF.

(C) 2015 Published by MECS Publisher. Selection and/or peer review under responsibility of the Research Association of Modern Education and Computer Science

\section{Introduction}

Today computer technology has permeated every sphere of modern man. The TV programs to the satellite launching the respective computer technologies are used. The security measure using the computer is the new trend. The IT network with their technologies are used in research work for the surveillance of the systems. The surveillance of different times and reasons is a new trend these days. This is a future research topic. Thus we have tried to make curbing software. This means this software has two major parts, event generation and response to the event. The event is generated that will check or restrain some feed input .This generated event will give response to stimuli by automatically sending a SMS to given destination. Thus, it is quite clear of

* Corresponding author. Tel.:

E-mail address: 
using two technologies as, c \# [5] and GSM. The event generation and procedure to interface the modem and the procedure to send a SMS are done with the c\# in the coded form. Then the actual sending of SMS is done with the GSM technology. This sends the message to the respective numbers. The gist is the event generation and the response by sending the text is the main purpose of the curbing software.

$\mathrm{C \#}$ (c sharp) is a computer language. It is a multi paradigm programming language encompassing imperative, functional, generic, object-oriented and component oriented programming discipline. It was developed by the Microsoft within the .NET developed team lead by the Anders Hejlsberg.GSM (global system for mobile communication) is the popular standard for the mobile telephony. This is the technology used for the mobile communication. They have their own frequency bands to communicate with the respective groups for the medium without interference.

The rest of the paper has been categorized as follows: Section 2 contains related work, Section 3 consists of the proposed system, Section 4 involves the system implementation, and Section 5 includes conclusion.

\section{Related Work}

In this section we have presented a number of previous related research works. In [12] Monitoring of Systems is done with the help of GSM technology. In [1] a significant number of secure systems are concerned with monitoring the environment. The most obvious example shown was the burglar alarm. Then there were meters for measuring consumption of utilities such as gas and electricity. There were also vehicle systems, such as missile telemetry, taximeters, and tachographs (devices used in Europe to record the speed and working hours of truck and bus drivers). In [2] the situation was addressed by categorizing monitoring systems based on their goals of detection and the time constraints of operation. This categorization was used to clarify what information is needed to perform detection as well as how the audit system should be structured to supply it in an appropriate manner. A prototype audit source was designed and constructed based on the information from the categorization. This audit system supplies information based on the type of detection to be performed. The new audit source was compared against an existing OS audit source and shown to have less overhead in many instances, generate a smaller volume of data, and generate useful information not currently available. In [3] "a study was done, the purpose of which was to improve the computer security auditing and surveillance capability of the customer's systems. In the Paper "A Novel Scheme for WSN-Based Security System: Detection and its Curb" [4] a method has been proposed for an automatic security system based on latest technology and have shown a means of integrating devices, software controlled for physical intrusion detection. The valuables with this idea of security system that will let secure minds preventing any non acceptable security prone event to happen at homes, shopping malls, banks etc can be protected. In [4] It has also been explored a model suitable for business and residential sectors to improve the standards and quality of security systems. [6] used remote control system using DTMF (Dual tone multi-frequency) signals which employed the use of telephone lines. The system consisted of two systems: The remote control system (the caller phone) and the phone monitoring system (the receiver system). The remote control system used the dual tone multifrequency signals to control the operation of the various appliances connected to the phone monitoring system. The devices connected to the phone monitoring system are controlled by predetermined commands. In [10] it has been shown that prevention of automobiles from theft can be done by using authorization for the owners and by building anti theft system in vehicles. Also security system face recognition is used for identifying the authorized person and the comparison is done through the preloaded faces for authorization. The vehicle will start only when the authorized person's face is recognized by the system. If the unauthorized person or theft attempts to drive the vehicle, an SMS/MMS will be sent to the owner along with the current location of the vehicle using GSM/GPS modem. IN [11] Intelligent Power Theft Detection System with instantaneous SMS alert has been developed which contained an Intelligent Statistical Meter placed at the transformer end or on electric high pole. It communicated with the consumer meters linked with it through RF and GSM technologies. It monitors and continuously sums up the energy consumed by the various consumer meters in a designated region and sets it as a reference value. Whenever this reference value is exceeded, an unmetered load or power 
theft is detected. An SMS is sent to the concerned company for necessary action.

\section{Proposed System}

In this section we have explained our system architecture, design and working mechanism of our proposed system. There are always some boundaries related to the concept of the system called as "GSM Based Monitoring and Control Systems", provides enhanced feature that can be used in commercial purposes, like offices having confidential material (trade secrets).In PC's or laptops any infiltration to the confidential material, unauthorized person can be reported to the owner of the material, monitoring the working of devices. This could be done by using proper settings and the use of this kind of system. This can be even used in the automation in the education systems. The GSM technology used in this concept is boundless as every other person is pocketed with the same. Thus to establish the security and the automation using this technology has the covered the scope on the whole. In this section we have explained our system architecture, design and working mechanism of our proposed system.

\subsection{System Architecture}

There are five modules in this system: GSM Module, SMS Messaging Module, DTMF Module, Database Module, and detailed working mechanism.

\subsubsection{GSM Module}

GSM (global system for mobile communication) is the popular standard for the mobile telephony. This is the technology used for the mobile communication. They have their own frequency bands to communicate with the respective groups for the medium without interference. In this paper more emphasis is given to understand the details of the existing GSM Technology, need and the benefits of monitoring existing systems remotely using GSM Technology.

\subsubsection{SMS Messaging Module}

SMS stands for Short Message Service [6]. It is a technology that enables the sending and receiving of messages between mobile phones. As suggested by the name "Short Message Service", the data that can be held by an SMS message is very limited. One SMS message can contain at most 140 bytes (1120 bits) of data, so one SMS message can contain up to 160 characters. One major advantage of SMS is that it is supported by $100 \%$ GSM mobile phones. Almost all subscription plans provided by wireless carriers include inexpensive SMS messaging service. The System can inform the security agencies immediately as the incident is going to happen and the incident could be monitored on the Mobile phones and even could be recorded.

\subsubsection{DTMF Module}

Another module is the DTMF [7] Module that has been implemented to monitor electrical devices remotely and even can be managed using DTMF immediately.

\subsubsection{AT Commands Module}

AT commands [8], [9] are instructions used to control a modem. AT is the abbreviation of ATtention. Every command line starts with "AT" or "at". That's why modem commands are called AT commands. There are two types of AT commands. Basic commands are AT commands that do not start with "+". For example, D (Dial), A (Answer), H (Hook control) and O (Return to online data state) are basic commands. Extended commands 
are AT commands that start with "+". All GSM AT commands are extended commands. For example, +CMGS (Send SMS message), +CMGL (List SMS messages) and +CMGR (Read SMS messages) are extended commands. Below are the SMS AT commands that may output the final result code +CMS ERROR:

$\begin{array}{ll}\text { +CRES (command name in text: Restore Settings) } & \text { +CMGD (command name in text: Delete Message) } \\ \text { +CMGL (command name in text: List Messages) } & \text { +CMGR (command name in text: Read Message) } \\ \text { +CMGS (command name in text: Sending Message) } & \text { +CSAS (command name in text: Save Settings) }\end{array}$

\subsubsection{Database Module}

An another application of the system is that it can be implemented in the offices where employees can send messages from and to the system, in such a case all the messages would be stored in the database of the office, thus providing the record of all the conversions.

\subsubsection{Working Mechanism}

Architecture of the overall system has been shown in figure 1. In this system the connect method is called in the form load method and when the user clicks on the connect button of connection setting tab. It creates an object of IO Port, sets its parameters and opens the Port. The method Panel1 Mouse Click () is executed when the user clicks the Pane2 which has YELLOW Background on the form. This method fetches the all mobile numbers from the database by executing SQL Command and stores the data in the Data Table, calls the method GetPDUstring and Senddata of the PDUencoder \& sendSMS Classes respectively. It sends the SMS to the recipients by calling sendSMS method. The method Panel2 MouseHover () is called when the moves towards the Panel2 which has RED Background color on the form. This method fetch the mobile numbers from the database and Will send the message to all the recipients. The method Add button click () has to add (insert) the data i,e the details of the person to the database by Validating the format of ID Field which should be Integer, making check weather ID is unique or not because ID is the Primary Key in the Table, Checking weather all the mandatory fields are supplied or not and by inserting the data in the table contact. The method Modify button click () is to update the details of the person(s) this updating is done on the basis of the Primary Key ID. The method Delete button click is to delete the record permanently from the database. The New record () will clear the input textboxes if they have any data so that the new information would be supplied and saved by clicking Save button. The method Grid mouse click () is to select any record present in the datagrid for deletion or for updating. The selected record is displayed in the input textboxes of the tab "contact details". The method Display button click () when invoked would fetch all the details of contact persons and will display them in the data grid of the Contact Details tab. The method Connect button click () is invoked when the user clicks the Connect button of the Connection Setting Tab. The purpose of this method is to make connection of the System to the GSM Modem or Mobile which supports AT commands. After making the connection it will change the connect button to disable form and will make disconnect button enabled.

The method Disconnect button () is called when the user clicks on the Disconnect button of the Connection Setting Tab. When executed this method will disconnect the system which was in the connected state. After disconnecting the connection it will make connecting button enable and disconnect button will be disabled. The method sendData () is called from the main form and it takes two arguments. Port and Message in the PDU format (in the hex form and includes both message and the recipient mobile number. This method will send AT COMMANDS to the IO Port. The method getPDUstring () is called from the main form this method calls four method of the same class namely stringtoseptet (), septettoOctet (), octettohex () and createPDU () and will return the PDU format of the message and destination number. The method Stringtoseptet () takes an string argument and converts the same to the septe form. The method Septettooctet () works on the message in the septet form and creates octet of the same. The method Octettohex () is to create the hexa form of the message which is in the octet form. The method CreatePDU () is to create the PDU format of the message and the 
mobile number here the former is in the hexa form and the lateral in the decimal half octet form. The method Decimal half octet () takes destination mobile number as an argument and converts the same to the decimal octet notation.

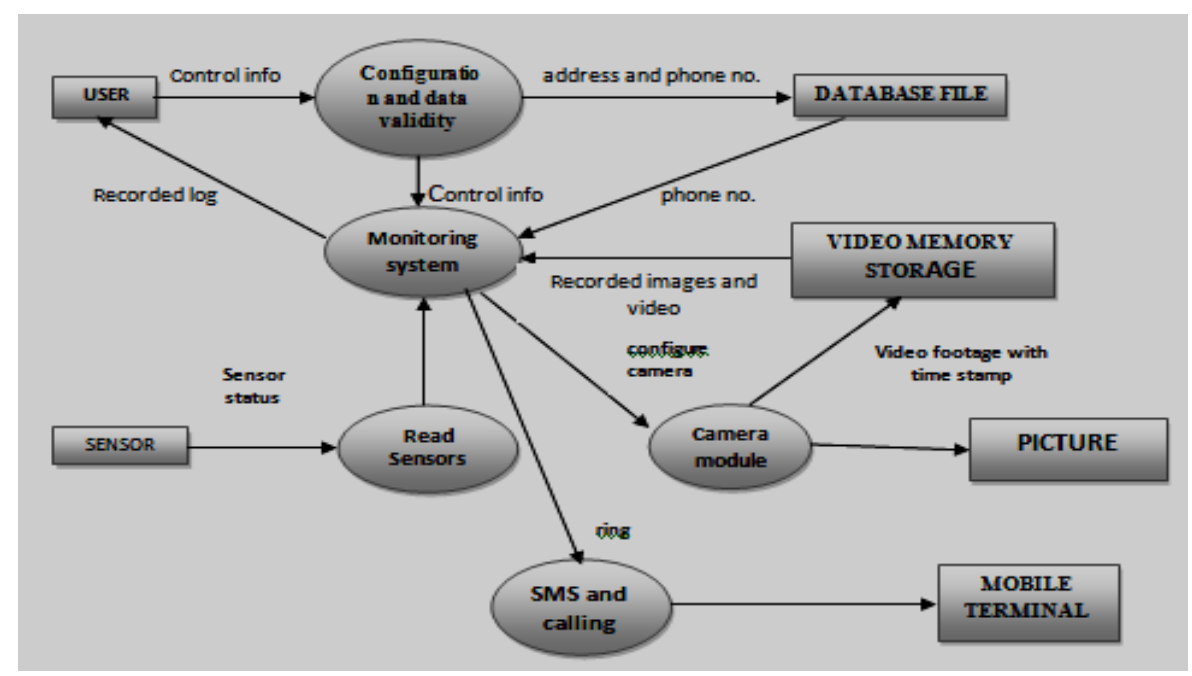

Fig.1. Architectural Diagram

\section{System Implementation}

This section consists of hardware and software implementation of our system.

\subsection{Hardware Implementation}

This part consists of some pictures of our proposed system. In this system we have used 3 IR receivers, 3 TSOP transmitters, DTMF, resistors, leds and Nokia phone as GSM modem. The working of our proposed system has been explained in section 3 .

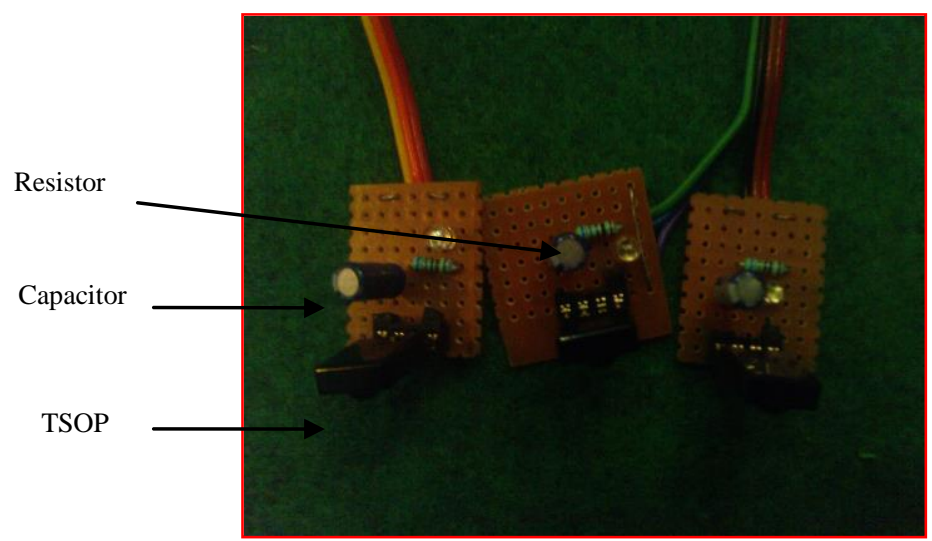

Fig.2. Three Receiver circuit picture 


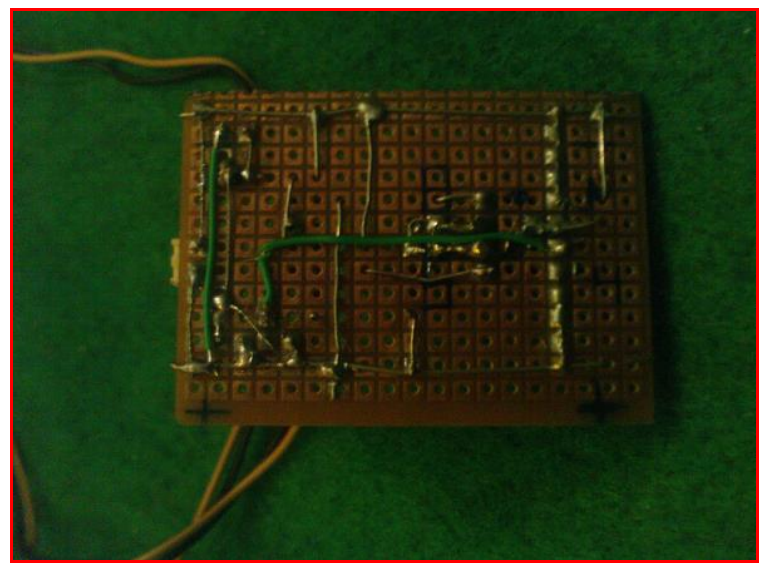

Fig.3. Soldering on Back side of general PCB

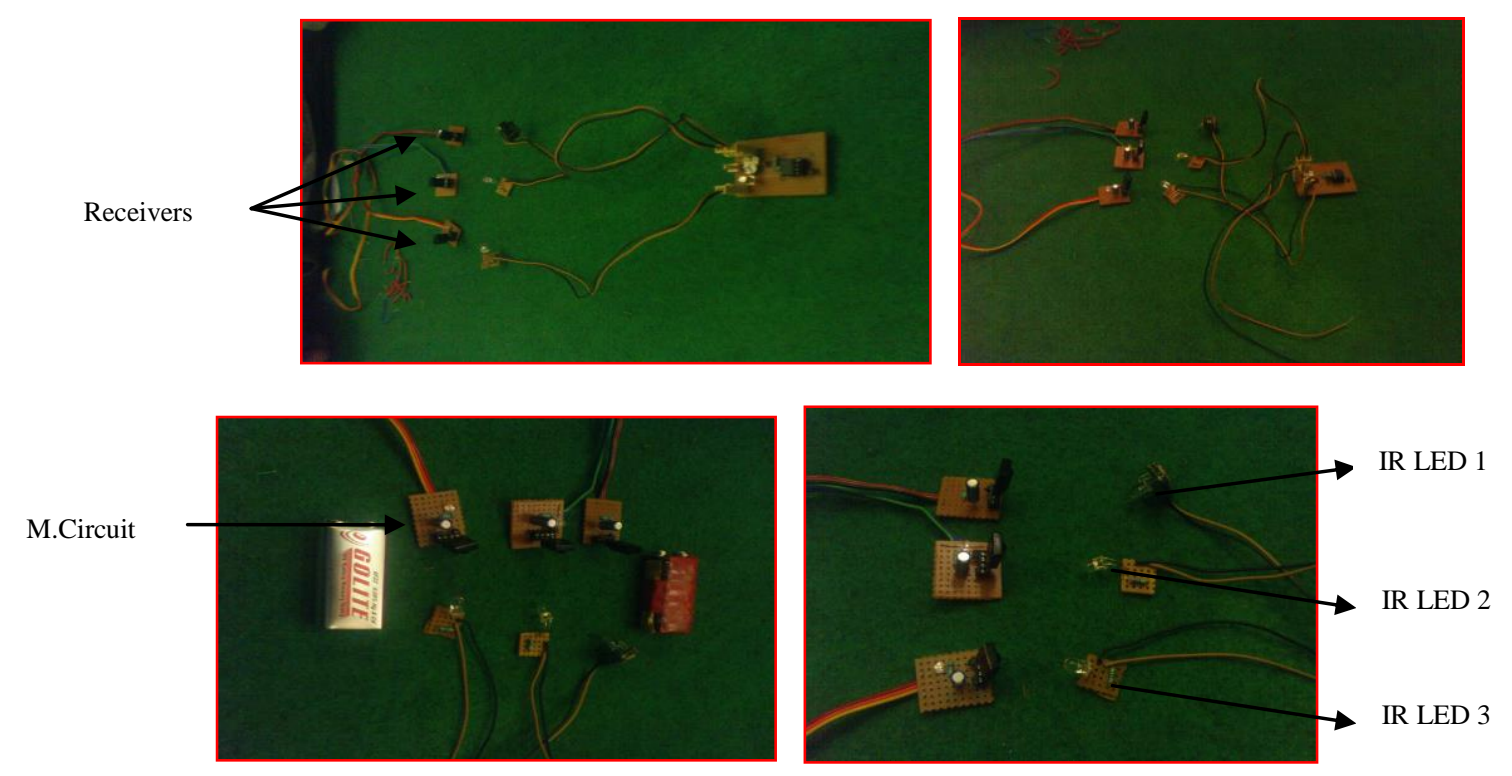

Fig.4. Signal Transmission and Reception

\subsection{Software Implementation}

The system that we used to control the whole process has been written in c\# on windows platform. The screen shots have been shown in figures 5, 6, 7, 8, 9, 10, and 11. Fig. 5 depicts about the interface, from where the demo starts. In fig. 6 we have set the port for the connection, namely com3. Thus, if any other port is being used then error occurs. 


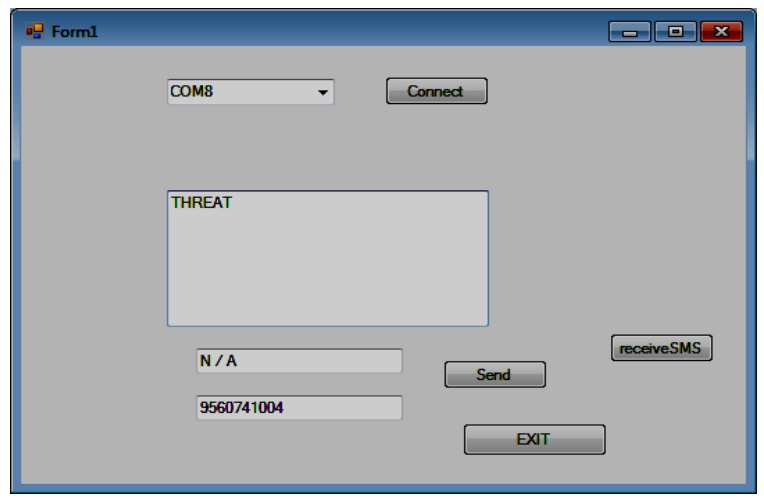

Fig.5. Interface from where demo starts

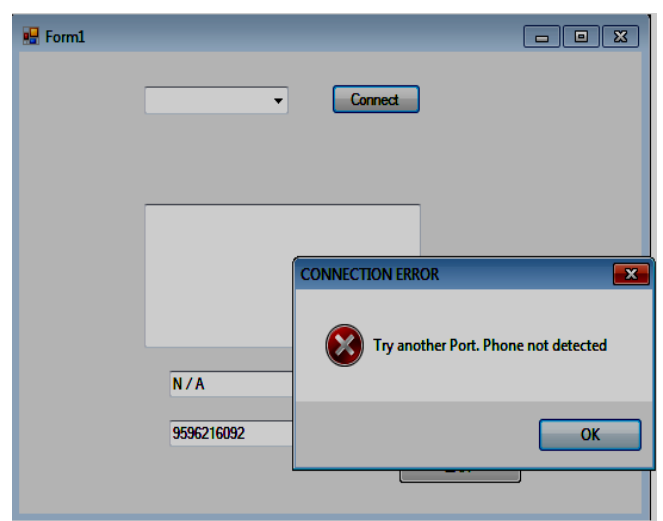

Fig.6. Interface where wrong port is given and error has been shown

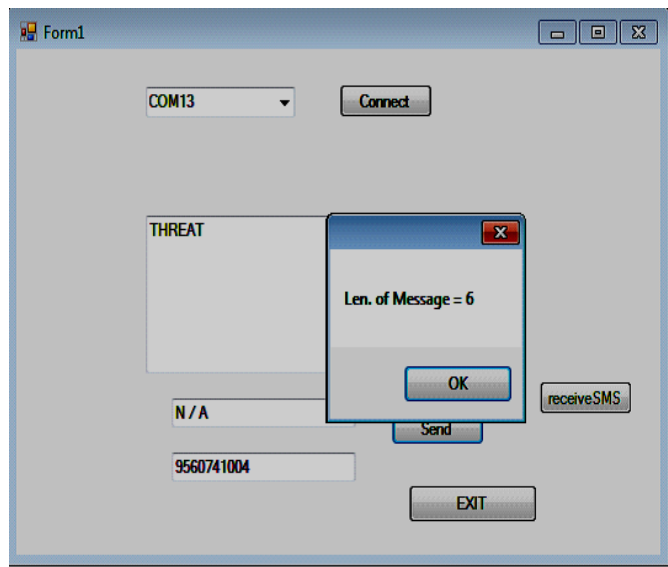

Fig.8. Interface which shows length of message

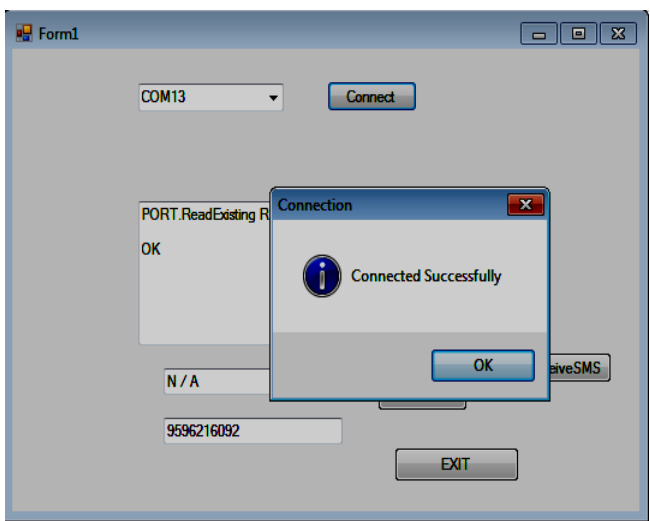

Fig.7. Interface which shows successful Connection of Com Port

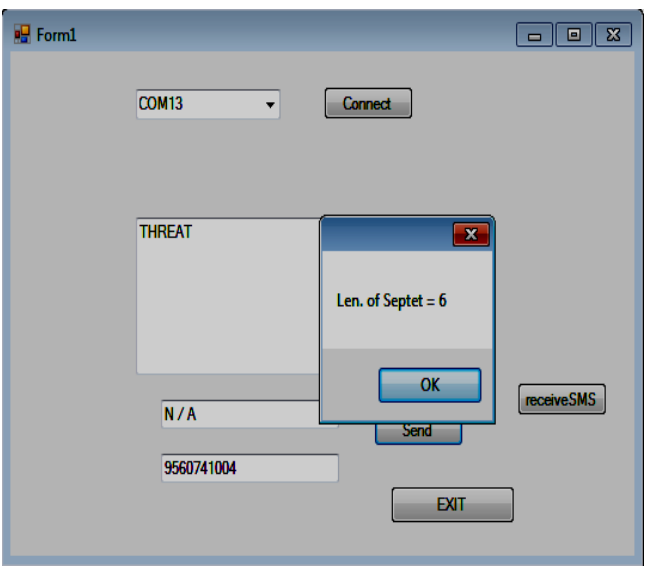

Fig.9. Interface which shows length of septet Port 


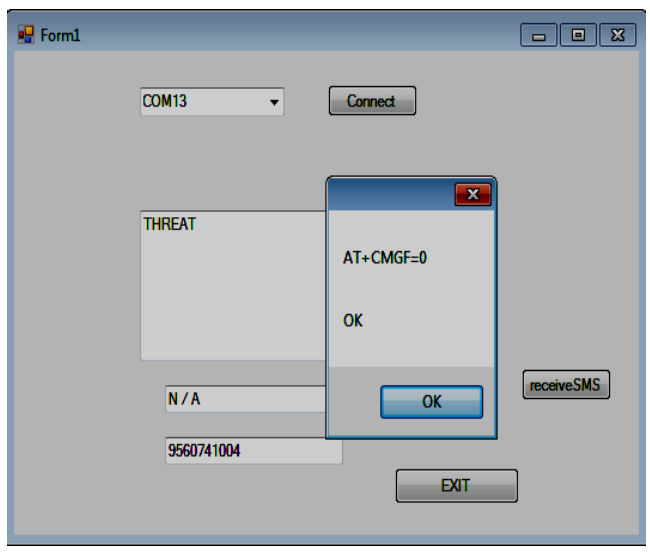

Fig.10. Interface which shows system is supported by GSM Modem

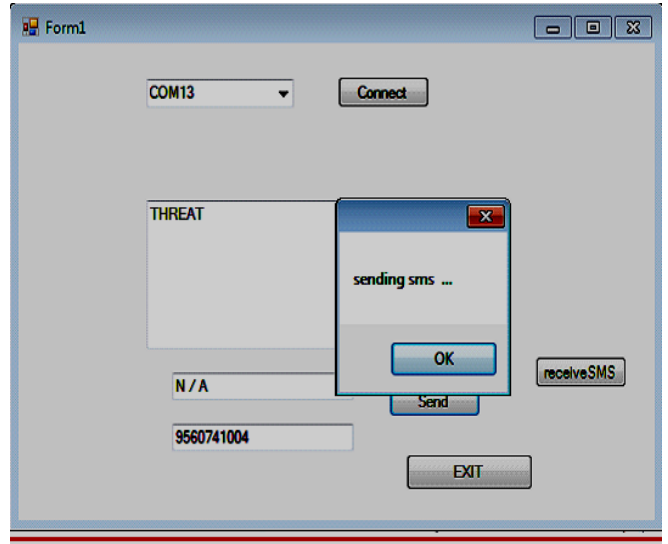

Fig.11. Interface where sending of SMS is done has been shown

In fig 7 we have given correct port number and the connection is successfully connected .In fig 8 the length of the message is calculated. In fig 9 the length of septet is calculated. In fig 10 it has been checked whether this system is supported by GSM modem or not. Fig 11 it has been shown the final step in which sending of sms is done.

\section{Conclusion}

The GSM Based Monitoring and Control of Systems can monitor \& even manage any kind system from any corner of them world which by combining two technologies - GSM Technology and the Dot Net technology. We have proposed a software and hardware based system to provide enhanced feature that can be used in commercial purposes, like offices having confidential material (trade secrets).In PC's or laptops any infiltration to the confidential material, unauthorized person can be reported to the owner of the material ,monitoring the working of devices. This could be done by using proper settings and the use of this kind of software. This can be even used in the automation in the education systems. The GSM technology used in this concept is boundless as every other person is pocketed with the same. Thus to establish the security and the automation using this technology has the covered the scope on the whole. In future we will it for the online detection; this can be done by using IP cameras to check the working (safety) of the system any time (provided the organization has internet).The hardware used in the same project with the sensing capabilities can be used in the malls, shops, security offices etc. for providing the cheap and safe security systems.

\section{References}

[1] Chapter 10 of Monitoring System (http://www.cl.cam.ac.uk/ rja14/Papers/SE-10.pdf).

[2] Kuperman, Benjamin A. "A Categorization of Computer Security Monitoring Systems and the Impact on the Design of Audit Sources”. Ph.D. Thesis, Purdue University, August, 2004.

[3] James P. Anderson Co. "Computer Security Threat Monitoring and Surveillance" Box 42 Fort Washington, Pa. 19034215 646-4706.

[4] Saima Maqbool, Nadia Yousuf, Afiya Tariq, Nafaja Qureshi “A Novel Scheme for WSN-Based Security System: Detection and its Curb" has not published yet.

[5] C \# step by step (Microsoft Press) and C\# 2005 for beginners (Wrox Publication).

[6] SMS tutorial for at command operations.

[7] Ogundele, Oladimeji Emmanuel "Design and Construction of DTMF based Home Automation System" A 
Project submitted to the department of electrical/computer engineering. Federal University of Technology, Minna, 2010.

[8] http://www.developershome.com/sms/howToUseHyperTerminal.asp and http://h0w2.blogspot.com/sms/How-to-enable-hyper-terminal-in-windows.html.

[9] http://help.expedient.com/dialup/hyperterminal.shtml and http://www.hilgraeve.com/hyperterminal-trial/.

[10] Mr. Raj Rai, Prof. Dinesh Katole, Miss. Nayan Rai, "Survey paper on Vehicle Theft Detection through Face Recognition System" in International Journal of Emerging Trends \& Technology in Computer Science (IJETTCS) Volume 3, Issue 1, January - February 2014 ISSN 2278-6856.

[11] B.O.Omijeh, G.I.Ighalo, F.I. Anyasi "Intelligent Power Theft Detection Model for Prepaid Energy Metering in Nigeria" in International Journal of Electronics Communication and Computer Engineering, in 2012 IJECCE.

[12] Saima Maqbool, Sajaad Ahmad Lone "Monitoring of System using GSM" International Journal of Computer Applications (0975 - 8887) Volume 117 - No.10, May 2015.

[13] Saima Maqbool, Nidhi Chandra, Shivraj Dagadi "ERPWS: An Energy Efficient Routing Protocol for Conductive Sensor based Water Level Monitoring and Control System using Zigbee and 74HC14 Inverter" In MECS 2013.

[14] Saima Maqbool, Nidhi Chandra" "Real Time Wireless Monitoring and Control of Water Systems using Zigbee 802.15.4”, In IEEE 2013.

\section{Authors' Profiles}

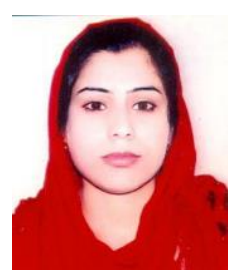

Saima Maqbool is an Assistant Professor at Islamic University of Science and Technology, Awantipura, Pulwama, Jammu \& Kashmir, India. She received M.Tech degree in Computer science and engineering from Amity University, Noida, India, B.E. (Computer Engineering) from Kashmir University, Jammu \& Kashmir. Her areas of interest are Network security and Wireless sensor networks.

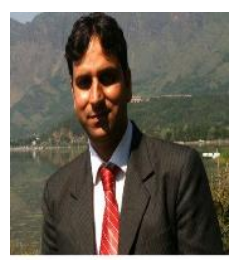

Sajaad Ahmad Lone is an Assistant Professor at working as an Assistant Professor at Islamic University of Science and Technology, Awantipura, Pulwama, Jammu \& Kashmir, India. He received M.Tech degree in information Technology from Guru Gobind Singh Inderprastha University, New Delhi, and currently pursuing PhD from National Institute of Technology Srinagar. The areas that interest him are mobile Adhoc networks, image processing. Network security, Adhoc and sensor networks and Ubiquitous Computing.

How to cite this paper: Saima Maqbool, Sajaad Ahmed Lone,"GSM based Monitoring and Control of Systems", IJWMT, vol.5, no.4, pp.15-23, 2015.DOI: 10.5815/ijwmt.2015.04.02 